\title{
BMJ Open Influential factors of postoperative pain trajectories in patients receiving intravenous patient-controlled analgesia: a single-centre cohort study in Taiwan
}

\author{
Ying-Hsuan Tai, ${ }^{1,2}$ Hsiang-Ling Wu, ${ }^{3,4}$ Shih-Pin Lin, ${ }^{3,4}$ Mei-Yung Tsou, ${ }^{3,4}$ \\ Kuang-Yi Chang (i) ${ }^{3,4}$
}

To cite: Tai Y-H, Wu H-L, Lin S-P, et al. Influential factors of postoperative pain trajectories in patients receiving intravenous patient-controlled analgesia: a single-centre cohort study in Taiwan. BMJ Open 2019;9:e031936. doi:10.1136/ bmjopen-2019-031936

- Prepublication history for this paper is available online. To view these files, please visit the journal online (http://dx.doi org/10.1136/bmjopen-2019031936).

Received 27 May 2019 Revised 14 October 2019 Accepted 21 October 2019

Check for updates

(C) Author(s) (or their employer(s)) 2019. Re-use permitted under CC BY-NC. No commercial re-use. See rights and permissions. Published by BMJ.

${ }^{1}$ Department of Anaesthesiology, Shuang Ho Hospital, Taipei Medical University, New Taipei City, Taiwan

${ }^{2}$ Department of Anaesthesiology, School of Medicine, College of Medicine, Taipei Medical University, Taipei, Taiwan ${ }^{3}$ Department of Anaesthesiology, Taipei Veterans General Hospital, Taipei, Taiwan

${ }^{4}$ School of Medicine, National Yang-Ming University, Taipei, Taiwan

Correspondence to Dr Kuang-Yi Chang; kychang@vghtpe.gov.tw

\section{ABSTRACT}

Objectives We aimed to investigate the factors associated with variations in postoperative pain trajectories over time in patients using intravenous patient-controlled analgesia (IV-PCA) for postoperative pain.

Design Retrospective cohort study.

Setting A single medical centre in Taiwan.

Participants Patients receiving IV-PCA after surgery.

Primary and secondary outcome measures Primary outcome was the postoperative pain scores.

Results A total of 3376 patients and 20838 pain score observations were analysed using latent curve models. Female and longer anaesthesia time increased the baseline level of pain ( $p=0.004$ and 0.003 , respectively), but abdominal surgery and body weight decreased it (both $p<0.001)$. Regarding the trend of pain resolution, lower abdominal surgery steepened the slope $(p<0.001)$; older age, American Society of Anesthesiologists (ASA) class $\geq 3$ and longer anaesthesia time tended to flatten the slope $(p<0.001,=0.019$ and $<0.001$, respectively). PCA settings did not affect the variations in postoperative pain trajectories.

Conclusions Patient demographics, ASA class, anaesthesia time and surgical sites worked together to affect postoperative pain trajectories in patients receiving IV-PCA. Latent curve models provided valuable information about the dynamic and complex relationships between the pain trajectories and their influential factors.

\section{INTRODUCTION}

Intravenous patient-controlled analgesia (IV-PCA) is a common and effective method to relieve postoperative pain. ${ }^{1}$ In IV-PCA, analgesic medication is self-administered by patients when pain is experienced, which optimises delivery of analgesics and minimises the interindividual variability in pharmacokinetics and pharmacodynamics. ${ }^{1}$ Compared with conventional as-needed analgesic regimens, IV-PCA offers better analgesia for postoperative pain and improves patient satisfaction. $^{2}$
Strengths and limitations of this study

- A large patient sample and numerous pain score observations were used to increase the statistical power and obtain precise and reliable results.

- The variations in postoperative pain trajectories over time were analysed as a whole, rather than separate comparisons of pain scores at distinct time points.

- Latent curve analyses provided a more comprehensive view to identify factors associated with the baseline and resolution of postoperative pain trajectories.

- Unmeasured confounders cannot be further assessed due to the retrospective design.

- The analgesic consumption was not further investigated.

A PCA pump can be programmed for several variables, including loading dose, demand dose, lockout interval and continuous infusion. Although the effectiveness of IV-PCA for acute postoperative pain is wellestablished, the effect of IV-PCA pump setting on analgesia has not been fully explored. Prior studies showed the addition of a continuous or background infusion to the demand dose for IV-PCA cannot provide analgesic benefits, and it only increases the analgesic consumption and the risk of postoperative adverse respiratory events in adult patients. ${ }^{3}$ However, few studies have ever evaluated the influential factors of postoperative pain trajectories in patients using IV-PCA in realworld routine practice.

The cause of postoperative pain is multifactorial, including patients' age, sex, and body weight and type of surgery. ${ }^{4-8}$ Besides, since pain may fluctuate in intensity and character over time, pain trajectory analysis is a better evaluation tool than individual 
pain measurements in reflecting the complex interplay between treatment and symptom. ${ }^{9} 10$ Studies have reported the association between postoperative pain trajectories and important clinical outcomes, including chronic postsurgical pain after knee arthroplasty, ${ }^{11}$ breast cancer surgery, ${ }^{12}$ and other types of surgery, ${ }^{1314}$ functional disabilities in arthritis patients, ${ }^{15}$ risk of postoperative readmission, ${ }^{16}$ and oncological outcomes of colorectal cancer. ${ }^{17} \mathrm{~A}$ recent post-hoc analysis of randomised trial showed the high pain cluster of pain trajectory significantly predicted prolonged postoperative pain and delayed opioid cessation, further highlighting the clinical implications of time course analysis of acute pain. ${ }^{18}$

To evaluate the effect of patient demographics, types of surgery, and different settings of IV-PCA pumps on postoperative analgesia, we conducted a retrospective cohort study at a tertiary medical centre and used latent curve analytical approaches to characterise postoperative pain trajectories. Specifically, multiple latent curve models were applied to determine the influential factors for baseline level and decrement trend of inhospital acute pain following major surgery. A clinical prediction model was further developed to offer a qualitative insight for the changes in postoperative pain. In this study, we hypothesised that patients' demographics, types of surgery, anaesthesia time, and pump settings of IV-PCA were associated with postoperative pain level and resolution rate in surgical patients.

\section{METHODS}

\section{Setting and patient selection}

In the retrospective cohort study, we reviewed the electronic medical records and collected the data of patients aged between 15 and 90 years undergoing spine, upper abdominal, lower abdominal or extremity surgery and receiving general anaesthesia with a postoperative use of IV-PCA for pain control at this centre in 2012. Patients were excluded from the analysis if they had regional anaesthesia or analgesia, the use of IV-PCA for less than 48 hours, inability to report pain intensity, $<2$ postoperative pain assessments within 7 days after surgery, postoperative ventilator support or intensive care beyond 24 hours, or surgical or anaesthetic complications that required an escalation in the acuity of their care.

\section{Anaesthesia and analgesia management}

All patients in the study received propofol $1-2 \mathrm{mg} \mathrm{kg}^{-1}$ and fentanyl $1-2 \mu \mathrm{g} \mathrm{kg}^{-1}$ for induction of general anaesthesia. Rocuronium or cis-atracurium was used to facilitate endotracheal intubation and repeated doses were given during surgery for muscle relaxation. Anaesthesia was maintained using sevoflurane $2-3$ vol\% or desflurane $6-8 \mathrm{vol} \%$ in a mixture of oxygen and air.

After the end of surgery, patients were transferred to a postanaesthetic care unit where IV-PCA was initiated. At this centre, contraindications to IV-PCA included no reasonable level of consciousness or cognitive function and need for postoperative ventilator support or intensive care beyond 24hours. A PCA infusion pump (Gemstar Yellow, Hospira, Chicago, Illinois, USA) was used to deliver morphine sulfate $1 \mathrm{mg} \mathrm{mL}^{-1}$ in normal saline, as reported in our previous studies. ${ }^{78}$ Patients were educated about using the PCA pump correctly both before and after surgery. All patients received an intravenous bolus of morphine $0.05 \mathrm{mg} \mathrm{kg}^{-1}$ as a loading dose for immediate pain relief on arrival at the postanaesthetic care unit. All PCA pumps were set to deliver a demand dose between 0.5 and $1.5 \mathrm{~mL}$, a lockout interval between 5 and $10 \mathrm{~min}$, and a continual basal infusion with a rate between 0 and $1.2 \mathrm{~mL} \mathrm{hour}^{-1}$ by anaesthesiologists in charge. IV-PCA was typically used for 48-72 hours after surgery and switched to oral acetaminophen or non-steroidal anti-inflammatory drugs thereafter. PCA pumps may be withdrawn earlier due to severe adverse effects of opioids (eg, nauseas, vomiting and sedation) in spite of adjusting the pump settings. The pain service team followed the response of patients receiving IV-PCA on a daily basis and recorded the pump setting at the end of the third day during the study period.

\section{Pain assessment and data acquisition}

Acute pain was assessed both at rest and during movement with self-report numeric rating scale (NRS) using an 11-point scale with response options from 'no pain' to 'the worst pain' by trained specialist nurses at 8-hour intervals for 24 hours after operation, 12-hour for 4 days and daily thereafter. The mean of daily NRS pain scores was retrieved in the first postoperative week from the electronic medical databank and served as the endpoints of the following latent curve analysis. Other collected covariates included patient's age, sex, body weight and height, body mass index, American Society of Anesthesiologists (ASA) class, anaesthesia time, and surgical site (ie, extremity, upper abdomen, lower abdomen and spine). Medical records of the enrolled patients were extracted by a specialist anaesthesiologist who were not involved in data analysis. Random samples of the extracted data were thoroughly checked by the authors to ensure the quality of data.

\section{Statistical analysis}

Patient characteristics and mean daily pain scores during the first postoperative week were expressed as mean \pm SD or count with percentage. Latent curve model was used to characterise the changes in daily average pain scores over time and evaluate how patient and surgery factors affected the trajectory of pain intensity. Three types of latent curve model, the basic, single predictor and multiple predictor models were applied to explore the transition of daily pain scores. ${ }^{19} 20$ The basic model was applied to estimate the baseline intercept and slope parameters, and then the single predictor model was used to evaluate the univariate relationship between collected variables and the intercept or slope parameter. A backward model selection strategy with the exit 
Table 1 Patient characteristics, PCA pump settings and postoperative pain scores

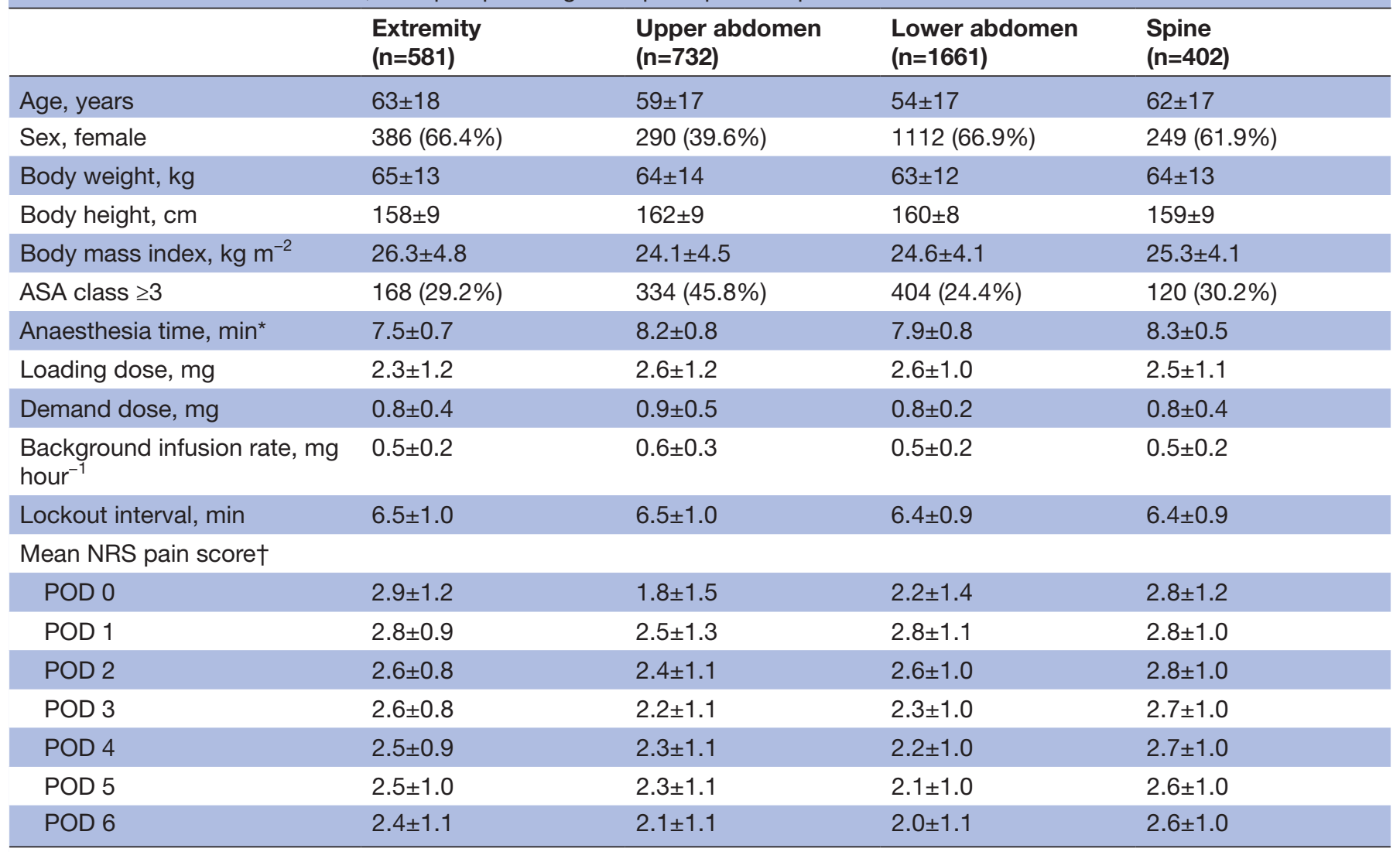

Values were mean \pm SD or counts (percent).

${ }^{*}$ On base-2 logarithmic scale.

†Number of pain score observations analysed in each postoperative day: POD 0: 3122 (92.5\%); POD 1: 3277 (97.1\%); POD 2: 3269 (96.8\%); POD 3: 3235 (95.8\%); POD 4: 3003 (89.0\%); POD 5: 2702 (80.0\%); POD 6: 2230 (66.1\%).

ASA, American Society of Anesthesiologists; NRS, numeric rating scale; PCA, patient controlled analgesia; POD, postoperative day.

criteria of $\mathrm{p}$ values greater than 0.05 was performed to identify independent explanatory factors of the intercept and slope parameters and determine the final multiple predictor model. The details of statistical technique on latent curve model refer to the previous literature. ${ }^{21} 22$ The goodness of fit was assessed using root mean square error of approximation (RMSEA) and comparative fit index $(\mathrm{CFI})$, and the values of RMSEA $<0.1$ and CFI $>0.9$ implied acceptable fit to data. ${ }^{23}$ All latent curve analyses were implemented using AMOS V.18.0 (SPSS, Chicago, Illinois, USA). Other statistical analyses were conducted with the SPSS V.18.0.

\section{Patient and public involvement}

This study is a retrospective analysis using the institutional medical database. There was no patient involved in the recruitment to and conduct of the study.

\section{RESULTS}

Patient characteristics are presented in table 1. There were 3376 patients and 20838 pain score observations included in the analysis. The proportions of enrolled patients undergoing extremity, upper abdominal, lower abdominal and spine surgeries were $17.2 \%, 21.7 \%, 49.2 \%$ and $11.9 \%$, respectively. The mean pain score was 2.3 on the postoperative day (POD) 0 , peaked at 2.7 on the POD 1 and then decreased consecutively to 2.2 on the POD 6 .

In the basic latent curve analysis, the estimated factor loading of slope parameters (a, b, ..., e in figure 1) from the POD 1 to POD 5 ranged from 1.44 to 0.05 . Note that the slope parameter of the POD 5 was not significant, which means there was no significant difference in mean pain scores between the POD 5 and 6 . The estimated values of intercept and slope parameters of the basic latent curve model were 2.14 and 0.36 , respectively. Accordingly, the estimated daily mean pain score during the POD 0 and 6 can be calculated as follows:

POD $0: 2.14+(1) 0.36=2.5$, POD $1: 2.14+(1.44) 0.36=2.64$,

Figure 2 presents the observed mean pain score with its SD bars and predicted value from latent curve analysis for each POD. The maximal difference between the predicted and observed daily mean pain score was less than 0.2 unit of NRS (on the POD 0 ).

Table 2 shows the result of the single predictor latent curve analysis. Among them, only demand dose and lockout interval did not exert any significant effect on the 


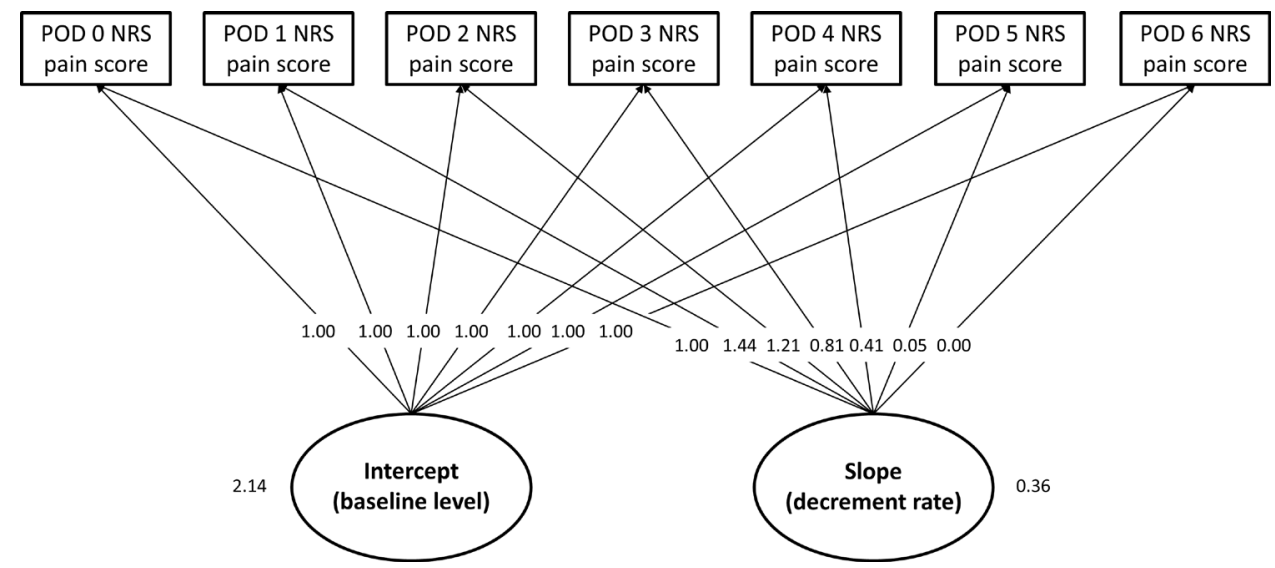

Figure 1 Basic model of latent curve analysis for postoperative pain scores. NRS, numeric rating scale; POD, postoperative day.

intercept or slope parameter of longitudinal pain scores. Other variables had significant effects on either intercept or slope parameter, or both.

Table 3 illustrates the result of multiple predictor latent curve analysis after model selection. Four independent predictors for the intercept parameter of mean pain scores were identified. Female and longer anaesthesia time were significantly associated with higher baseline pain scores. With respect to the surgical site, patients undergoing upper or lower abdominal surgeries had lower baseline pain scores than those undergoing extremity surgeries. Furthermore, patients with higher body weight tended to have lower baseline NRS pain scores. Regarding the decrement slope of pain scores in subsequent days, patients with older age, ASA class $\geq 3$ and longer anaesthesia time had a slower pain resolution; contrarily, those undergoing lower abdominal surgeries had a faster pain resolution compared with those undergoing extremity surgeries. The RMSEA and CFI values of the final model were 0.077 and 0.897 , respectively, and its graphic presentation is illustrated in figure 3 .

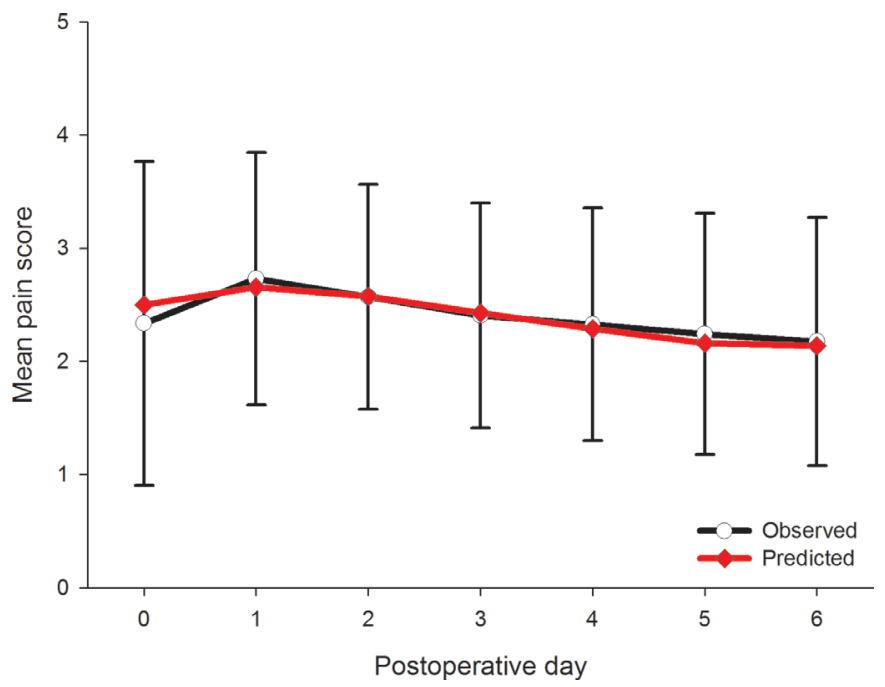

Figure 2 The observed mean pain score with its SD bars and predicted value from latent curve analysis for each postoperative day.
Based on the results, the predicted pain score during the first postoperative week can be estimated without difficulty. For example, for a 50-year-old woman with body weight of $60 \mathrm{~kg}$ and ASA class 2, undergoing an upper abdominal surgery with anaesthesia time $180 \mathrm{~min}$, her estimated mean pain score on the POD 1 can be calculated with the following formula:

$\left[2.406+0.085 \times 1(\right.$ female $) \quad 0.004 \times 60($ bodyweight $)+0.054 \times \log _{2}(180)$

(anaesthesia time) $0.368 \times 1$ (upper abdominal surgery) $0.303 \times 0$

(lower abdominal surgery) $]+2.63 \times[(0.384-0.002 \times 50($ age $)$

$\left.0.028 \times \log _{2}(180)\right)($ anaesthesia time $) 0.023 \times 0($ ASA class 3$)$

$+0.068 \times 0$ (lower abdominal surgery $)]=2.48$.

The predicted mean pain scores of other PODs can also be estimated in a similar manner.

\section{DISCUSSION}

In the present study, latent curve analyses were used to model the longitudinal pain scores after major surgery and to evaluate potential influential factors of postoperative pain trajectory. Our results showed that patient and surgery factors rather than PCA pump settings exert significant effects on acute pain trajectory in patients receiving IV-PCA. In addition, although we developed a prediction model for the postoperative pain trajectory, this model would need to be validated before being used clinically. The latent curve analysis not only quantifies the pain intensity but also distinguishes between pain intensity and pain resolution over time. Our analysis was based on a large patient sample to increase the statistical power and obtain precise and reliable results.

\section{Analysis for time course of acute pain}

Compared with conventional one-time measurements of acute pain, modelling pain trajectories may substantially improve research on pain and optimise postoperative pain monitoring. Besides, analyses for temporal domain of pain revealed a meaningful link between psychosocial modulators of postoperative pain and pain chronification. ${ }^{14} \mathrm{~A}$ variety of analytical methods have been used to 
Table 2 Effects of selected variables on the intercept and slope parameters in the single predictor model

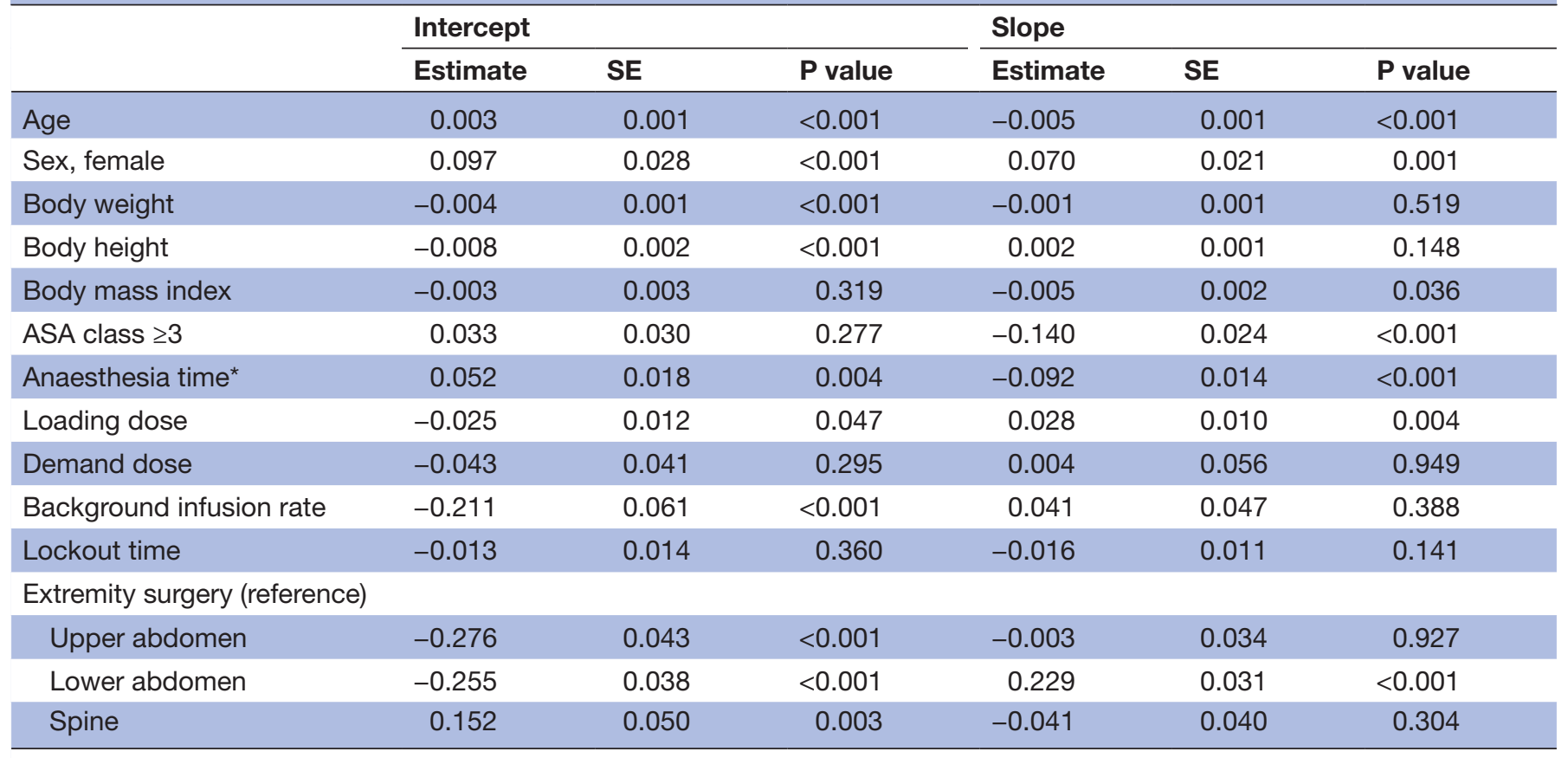

*On base-2 logarithmic scale.

ASA, American Society of Anesthesiologists.

assess the pain trajectory in previous studies, including latent curve model, ${ }^{9131425}$ Bayesian change-point model, ${ }^{25}$ and cluster analysis for trajectory classification. ${ }^{10} 12$ 16-18 Compared with change-point model and cluster analysis, latent curve model is useful in distinguishing between pain intensity and pain resolution and identifying potential influential factors for these two important parameters of pain.

\begin{tabular}{|c|c|c|c|}
\hline & Estimate & SE & $\begin{array}{l}P \\
\text { value }\end{array}$ \\
\hline \multicolumn{4}{|l|}{ Intercept } \\
\hline Sex (female vs male) & 0.085 & 0.030 & 0.004 \\
\hline Body weight & -0.004 & 0.001 & $<0.001$ \\
\hline Anaesthesia time* & 0.054 & 0.018 & 0.003 \\
\hline \multicolumn{4}{|c|}{ Extremity surgery (reference) } \\
\hline Upper abdomen & -0.368 & 0.039 & $<0.001$ \\
\hline Lower abdomen & -0.303 & 0.031 & $<0.001$ \\
\hline \multicolumn{4}{|l|}{ Slope } \\
\hline Age & -0.002 & 0.000 & $<0.001$ \\
\hline ASA class $\geq 3$ & -0.023 & 0.010 & 0.019 \\
\hline Anaesthesia time* ${ }^{*}$ & -0.028 & 0.007 & $<0.001$ \\
\hline \multicolumn{4}{|c|}{ Extremity surgery (reference) } \\
\hline Lower abdomen & 0.068 & 0.013 & $<0.001$ \\
\hline
\end{tabular}

${ }^{*}$ On base-2 logarithmic scale.

ASA, American Society of Anesthesiologists.

\section{IV-PCA pump settings}

The multiple latent curve model showed no significant effect of IV-PCA pump settings on the baseline level or decrement rate of postoperative pain after adjusting for patient and surgery factors, including the loading dose, demand dose, lockout interval and background infusion rate of morphine sulfate.

Although the optimal demand dose is uncertain, prior reports suggested that the optimal demand dose is $1 \mathrm{mg}$ for morphine in opioid-naive patients. ${ }^{1}$ However, our analysis did not show any dose-dependent association between demand dose and postoperative analgesia. Furthermore, the lockout interval may influence the analgesic efficacy and medication-related adverse events of IV-PCA, although the optimal lockout interval is unknown. ${ }^{1}$ Prior studies showed that varying the interval within 5-10 min appears to have no significant effect on analgesia or adverse effects, ${ }^{1}$ which agrees with our results. As to the effect of background infusion of IV-PCA on analgesia, previous studies showed that a background infusion provides no analgesic benefit in adult patients, in line with our results. ${ }^{3}$

\section{Age, sex and body weight}

Our analysis demonstrated that older age tended to flatten the decrement slope of postoperative pain intensity without affecting the baseline pain level. A prior study showed that older patients had a slightly slower resolution of postoperative pain than younger patients. ${ }^{4}$ It was likely that older patients had either lower pain sensitivity or better pain relief from analgesics compared with their younger counterparts. ${ }^{45}$ Besides, a prospective cohort 


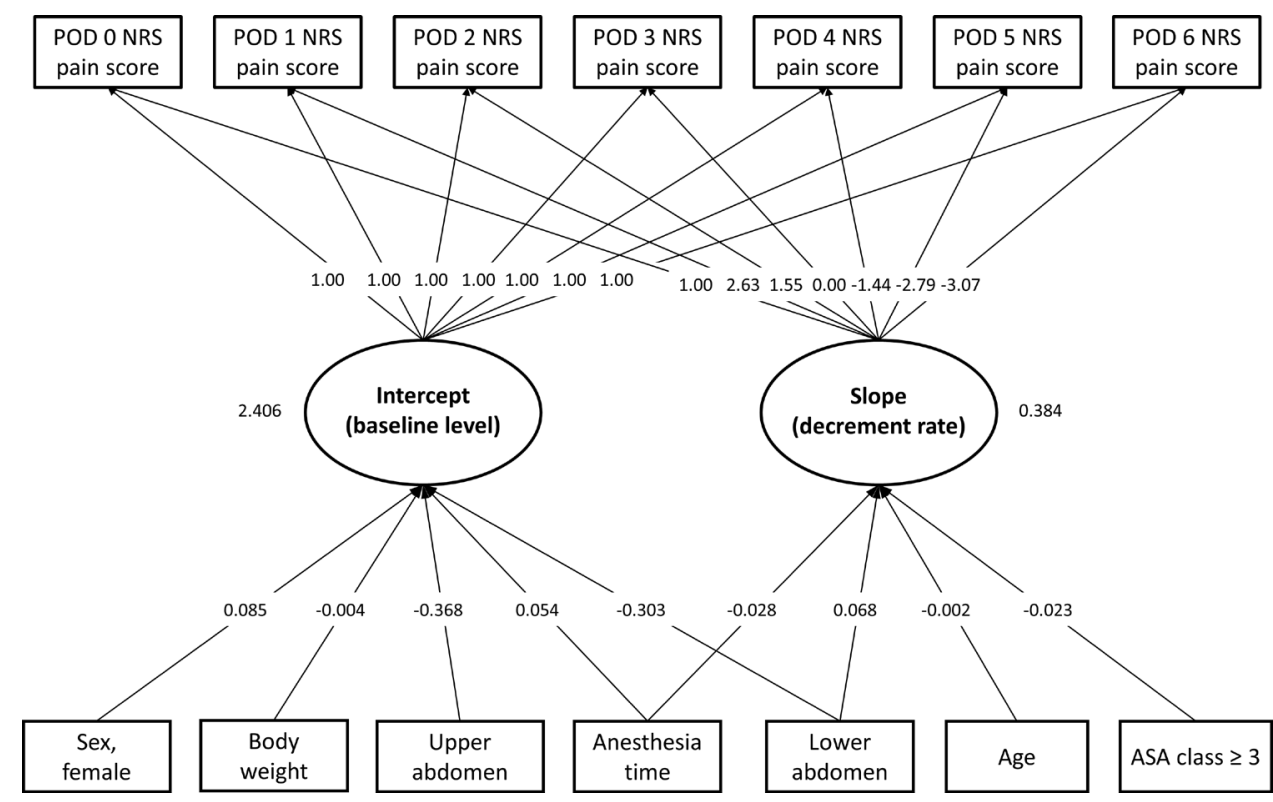

Figure 3 Final model of latent curve analysis for postoperative pain scores. NRS, numeric rating scale; POD, postoperative day.

study showed that older age was independently associated with lower postoperative pain intensity on the first POD ${ }^{6}$ contrasting with our results. The discrepant finding may be explained by the difference in patient characteristics, types of surgery and pain management.

In our study, female patients were found to have higher baseline pain scores than males after major surgery, consistent with some prior studies. ${ }^{46}{ }^{18}$ However, the sex difference in the postoperative pain intensity and analgesic consumption remained controversial in previous literature. A large-scale cohort study showed male patients had higher baseline postoperative pain scores and faster pain resolution compared with females, contrasting with our results. ${ }^{10}$ A systemic review showed a conflicting result for sex as a predictor for either postoperative pain or analgesic consumption, which could be due to the difference in patient characteristics and sample size among separate studies. ${ }^{5}$

Few studies have investigated the effect of body weight on postoperative pain and analgesic consumption, and the results remained conflicting and inconclusive. ${ }^{5}$ Of note, our analysis showed patients with higher body weight had lower baseline pain intensity after surgery. However, the effect size was relatively small, and the clinical significance of this finding should be interpreted with caution.

\section{Anaesthesia time and sites of surgery}

Patients undergoing surgeries with longer anaesthesia time were found to have higher baseline pain intensity and a slower pain resolution in our study, which may reflect the impact of complexity of surgical procedures and extent of surgical intervention on acute pain severity. Regarding the site of surgery, our analysis showed that compared with extremity surgeries, abdominal surgeries caused a lower baseline level of postoperative pain, and lower abdominal surgeries were correlated with a faster pain resolution during the hospital stay. In previous literature, the association between type of surgery and postoperative pain outcome was often interpreted only as a different subspecialty. Abdominal surgery, orthopaedic surgery, thoracic surgery and procedures involving a long duration of surgery may positively correlate with postoperative pain. ${ }^{5}$ However, the definition of types of surgery and difference in patient characteristics may affect the result of different studies, which should be interpreted in a clinical context.

\section{Clinical implication}

For clinical implications, particular attention should be paid to patients with a predicted high baseline level or a slow resolution of postoperative pain. To improve the pain control in these patients, pre-emptive and multimodal analgesia strategies should be considered instead of a postoperative use of IV-PCA alone. ${ }^{2627}$

\section{Limitation}

First, the patients were not randomised and the effects of unmeasured confounders cannot be further assessed, such as preoperative pain and opioid usage, psychological distress, intraoperative consumption of opioids and postoperative timing of rehabilitation. ${ }^{561418}$ Second, we used the numerical rating scale as a measurement tool of pain intensity, instead of analgesic consumption. Recent study has reported different trajectories between postoperative pain and opioid consumption. ${ }^{28}$ Although the validity and reliability of self-report pain rating scale have been verified in Chinese adults, ${ }^{29}$ biases may exist in some subpopulations. ${ }^{30}$ Third, we did not analyse the adverse events of analgesic medication (eg, respiratory depression), and unintended bolus and malfunction of IV-PCA due to data unavailability. Fourth, some patients might 
have very different postoperative pain trajectories and heterogeneity in the slopes, which awaits further investigation to explain unusual pain resolution after surgery. Finally, compared with previous studies, the pain scores of this study were relatively low, which may be explained by discrepancies in type of surgery, pain measurement (worst or average pain) and management, and race differences in pain sensitivity. ${ }^{9-18}$ Although we identified several factors statistically significantly associated with postoperative pain level and decrement rate, the effect size of some influential factors may be too small to have clinical significance.

In conclusion, patient demographics, ASA class, anaesthesia time, and surgical sites worked together to affect postoperative pain trajectories in patients receiving IV-PCA. Latent curve models provided valuable information about the dynamic and complex relationships between the pain trajectories and their influential factors. Our results offered practical information for clinicians to manage postoperative pain and enhance the quality of pain control.

Contributors Y-HT contributed to data acquisition and manuscript drafting. H-LW contributed to data verification. S-PL contributed to data acquisition. M-YT helped revise the manuscript. K-YC contributed to statistical analysis, manuscript revision and gave final approval of the version to be published and agreed to be accountable for ensuring the accuracy or integrity of the work. All authors read and approved the final manuscript.

Funding This study was supported by the grants from Taipei Veterans General Hospital, Taipei, Taiwan (V106C-048), Yen Tjing Ling Medical Foundation, Taipei, Taiwan (Cl-108-27), Anesthesiology Research and Development Foundation, Taipei, Taiwan (ARDF10804) and Ministry of Science and Technology, Taipei, Taiwan, ROC (MOST107-2511-H-075-001).

Competing interests None declared.

Patient consent for publication Written informed consent was waived.

Ethics approval The study was approved by the Institutional Review Board of Taipei Veterans General Hospital, Taipei, Taiwan (IRB-TPEVGH No. 2015-11-010CC).

Provenance and peer review Not commissioned; externally peer reviewed.

Data availability statement Data are available upon reasonable request.

Open access This is an open access article distributed in accordance with the Creative Commons Attribution Non Commercial (CC BY-NC 4.0) license, which permits others to distribute, remix, adapt, build upon this work non-commercially, and license their derivative works on different terms, provided the original work is properly cited, appropriate credit is given, any changes made indicated, and the use is non-commercial. See: http://creativecommons.org/licenses/by-nc/4.0/.

ORCID iD

Kuang-Yi Chang http://orcid.org/0000-0002-9062-5519

\section{REFERENCES}

1 Macintyre PE. Safety and efficacy of patient-controlled analgesia. $\mathrm{Br}$ $J$ Anaesth 2001;87:36-46.

2 Hudcova J, McNicol ED, Quah CS, et al. Patient controlled opioid analgesia versus conventional opioid analgesia for postoperative pain. Cochrane Database Syst Rev 2006:CD003348.

3 George JA, Lin EE, Hanna MN, et al. The effect of intravenous opioid patient-controlled analgesia with and without background infusion on respiratory depression: a meta-analysis. J Opioid Manag 2010;6:47-54.

4 Tighe PJ, Le-Wendling LT, Patel A, et al. Clinically derived early postoperative pain trajectories differ by age, sex, and type of surgery. Pain 2015;156:609-17.
5 HY I, Abrishami A, Peng Philip W, et al. Predictors of postoperative pain and analgesic consumption: a qualitative systematic review. Anesthesiology 2009;111:657-77.

6 Gerbershagen HJ, Pogatzki-Zahn E, Aduckathil S, et al. Procedurespecific risk factor analysis for the development of severe postoperative pain. Anesthesiology 2014;120:1237-45.

7 Lin S-P, Chang K-Y, Chen H-H, et al. Predicting procedure-specific morphine consumption of intravenous patient-controlled analgesia with random-effect model approach. Clin J Pain 2019;35:43-9.

8 Yen C-R, Tsou M-Y, Mandell MS, et al. An analysis of patient variables that influence intravenous patient-controlled analgesic use of morphine with quantile regression. Anesthesiology 2010;112:688-95.

9 Chapman CR, Donaldson GW, Davis JJ, et al. Improving individual measurement of postoperative pain: the pain trajectory. $J$ Pain 2011;12:257-62.

10 Kannampallil T, Galanter WL, Falck S, et al. Characterizing the pain score trajectories of hospitalized adult medical and surgical patients. Pain 2016;157:2739-46.

11 Lavand'homme PM, Grosu I, France M-N, et al. Pain trajectories identify patients at risk of persistent pain after knee arthroplasty: an observational study. Clin Orthop Relat Res 2014;472:1409-15.

12 Okamoto A, Yamasaki M, Yokota I, et al. Classification of acute pain trajectory after breast cancer surgery identifies patients at risk for persistent pain: a prospective observational study. J Pain Res 2018;11:2197-206.

13 Althaus A, Arránz Becker O, Moser K-H, et al. Postoperative pain trajectories and pain Chronification-an empirical typology of pain patients. Pain Med 2018;19:2536-45.

14 Althaus A, Arránz Becker O, Neugebauer E. Distinguishing between pain intensity and pain resolution: using acute postsurgical pain trajectories to predict chronic post-surgical pain. EJP 2014;18:513-21.

15 James RJE, Walsh DA, Ferguson E. Trajectories of pain predict disabilities affecting daily living in arthritis. Br J Health Psychol 2019;24:485-96.

16 Hernandez-Boussard T, Graham LA, Desai K, et al. The fifth vital sign: postoperative pain predicts 30 -day readmissions and subsequent emergency department visits. Ann Surg 2017;266:516-24.

17 Chang W-K, Tai Y-H, Lin S-P, et al. An investigation of the relationships between postoperative pain trajectories and outcomes after surgery for colorectal cancer. J Chin Med Assoc 2019. doi:10.1097/JCMA.0000000000000166. [Epub ahead of print: 331 Jul 2019].

18 Hah JM, Cramer E, Hilmoe H, et al. Factors associated with acute pain estimation, postoperative pain resolution, opioid cessation, and recovery: secondary analysis of a randomized clinical trial. JAMA Netw Open 2019;2:e190168.

$19 \mathrm{PH}$ L, Tsou MY, Chang KY. Modeling the trajectory of analgesic demand over time after total knee arthroplasty using the latent curve analysis. Clin J Pain 2015;31:776-81.

20 Chao P-W, Lin S-P, Tsou M-Y, et al. Assessing the impact of renal function on trajectory of intravenous patient-controlled analgesic demands over time after open and laparoscopic colorectal surgery using latent curve analysis. Clin J Pain 2016;32:695-701.

21 Bollen KA, Curran PJ. Latent curve models: a structural equation perspective. Hoboken, NJ: Wiley Blackwell, 2006.

22 Preacher KJ, Wichman AL, MacCallum RC, et al. Latent growth curve modeling. Los Angeles, CA: SAGE Publications, 2008.

23 Byrne BM. Structural equation modeling with AMOS: basic concepts, applications, and programming. New York, NY: Routledge, 2010.

24 Kline RB. Principles and practice of structural equation modeling. 3rd. New York, NY: Guilford Press, 2011.

25 Houle TT, Miller S, Lang JE, et al. Day-To-Day experience in resolution of pain after surgery. Pain 2017;158:2147-54.

26 Memtsoudis SG, Poeran J, Zubizarreta N, et al. Association of multimodal pain management strategies with perioperative outcomes and resource utilization. Anesthesiology 2018;128:891-902.

27 Kissin I, Analgesia P. Preemptive analgesia. Anesthesiology 2000;93:1138-43.

28 Pagé MG, Karanicolas PJ, Cleary S, et al. In-Hospital opioid consumption, but not pain intensity scores, predicts 6-month levels of pain catastrophizing following hepatic resection: a trajectory analysis. Eur J Pain 2019;23:503-14.

29 Li L, Liu X, Herr K. Postoperative pain intensity assessment: a comparison of four scales in Chinese adults. Pain Med 2007:8:223-34

30 Gagliese L, Katz J. Age differences in postoperative pain are scale dependent: a comparison of measures of pain intensity and quality in younger and older surgical patients. Pain 2003;103:11-20. 